\title{
Silent sinus syndrome: dynamic changes in the position of the orbital floor after restoration of normal sinus pressure
}

\author{
R SIVASUBRAMANIAM ${ }^{1}$, R SACKS $^{2}$, M THORNTON $^{3}$ \\ Ear, Nose and Throat Departments, ${ }^{1}$ The Canberra Hospital, Australian Capital Territory, ${ }^{2}$ Concord General \\ Hospital, New South Wales, Australia, and ${ }^{3}$ St Vincent's University Hospital, Dublin, Ireland
}

\begin{abstract}
Background: Silent sinus syndrome is characterised by spontaneous enophthalmos and hypoglobus, in association with chronic atelectasis of the maxillary sinus, and in the absence of signs or symptoms of intrinsic sinonasal inflammatory disease. Traditionally, correction of the enophthalmos involved reconstruction of the orbital floor, which was performed simultaneously with sinus surgery. Recently, there has been increasing evidence to support the performance of uncinectomy and antrostomy alone, then orbital floor reconstruction as a secondstage procedure if needed.

Methods: We performed a retrospective review of 23 cases of chronic maxillary atelectasis managed in our unit with endoscopic uncinectomy and antrostomy alone. All patients were operated upon by the same surgeon.

Results: Twenty-two of the 23 patients had either complete or partial resolution. One patient had ongoing enophthalmos, and was considered for an orbital floor reconstruction as a second-stage procedure.

Conclusion: Our case series demonstrates that dynamic changes in orbital floor position can occur after sinus reventilation. These findings support the approach of delaying orbital floor reconstruction in cases of silent sinus syndrome treated with sinus re-ventilation, as such reconstruction may prove unnecessary over time.
\end{abstract}

Key words: Maxillary Sinus; Pathology; Enophthalmos; Sinusitis; Surgical Procedures, Operative; Endoscopy

\section{Introduction}

Spontaneous enophthalmos and hypoglobus, in association with chronic atelectasis of the maxillary sinus, and in the absence of signs or symptoms of intrinsic sinonasal inflammatory disease, is a condition first described by Montgomery in 1964, and termed the silent sinus syndrome by Soparkar et al. in 1994. ${ }^{1,2}$ Maxillary sinus volume reduction, characterised radiologically by the inward bowing of one or more antral walls, develops as a result of osteomeatal occlusion. Over time, hypoventilation results in resorption of gases into the capillaries of the closed sinus cavity, creating negative pressure. This results in chronic subclinical inflammation, leading to maxillary atelectasis. ${ }^{3,4}$ This displaces the medial infundibular wall laterally; when such collapse is significant, the orbital volume increases, with ensuing enophthalmos and hypoglobus., 3,5,6

Silent sinus syndrome is a slow, progressive, unilateral disorder, with changes occurring over years. Most patients present in their third to fifth decade with no obvious preceding sinus symptoms. Examination usually shows orbital asymmetry, with deepening of the superior orbital sulcus and eyelid retraction. All patients have a degree of hypoglobus, ranging from 2 to $6 \mathrm{~mm}$, and enophthalmos, ranging from 2 to $5 \mathrm{~mm}$, according to one of the largest published series. ${ }^{2}$ Ocular motility and visual acuity are not always affected; occasionally, patients complain of diplopia. The mainstay of diagnosis also involves computed tomography scan of the paranasal sinuses.

Chronic maxillary atelectasis is another term used in the otolaryngology literature to describe spontaneous enophthalmos in association with a contracted ipsilateral maxillary sinus. ${ }^{7}$ Chronic maxillary atelectasis is divided into three types: type I, with a membranous deformity; type II, with a bony deformity; and type III, with clinical features. ${ }^{7}$ Although silent sinus syndrome can be considered a form of chronic maxillary atelectasis, and type III chronic maxillary atelectasis is similar to silent sinus syndrome, one of the main differences between them is the absence of nasal symptoms in the silent sinus syndrome patient. 
For years, endoscopic uncinectomy and middle meatal antrostomy have been fundamental to the management of such cases, exposing the sinus ostium and re-establishing sinus ventilation. Correction of associated enophthalmos involved the reconstruction of the orbital floor, which was usually performed via a transconjunctival approach, simultaneously with sinus surgery. ${ }^{8,9}$

However, in 2003 Thomas et al. noted a return to normal globe position in two of four patients following uncinectomy and antrostomy alone. ${ }^{10}$ This led them to recommend that orbital floor reconstruction be performed as a staged procedure for patients with persistent enophthalmos of greater than $2 \mathrm{~mm}$, or related visual disturbances, after reassessment at six months.

In our unit, we have successfully managed a number of cases of silent sinus syndrome with endoscopic uncinectomy and antrostomy. During follow up of these cases, we monitored the position of the orbital floor using nasal endoscopy. In the presented series, we demonstrate the dynamic changes in orbital floor position that can occur after sinus re-ventilation, supporting the principle of delayed orbital floor reconstruction in cases of silent sinus syndrome.

\section{Materials and methods}

We performed a retrospective review of the clinical records of all patients diagnosed with chronic maxillary atelectasis and silent sinus syndrome in our unit.

Our diagnostic criteria for chronic maxillary atelectasis was enophthalmos and/or hypoglobus, with sinus opacification noted on computed tomography or intra-operatively. Our diagnostic criteria for silent sinus syndrome included altered facial appearance, with enophthalmos and/or hypoglobus, computed tomography imaging showing a contracted ipsilateral maxillary sinus, and absence of sinusitis symptoms.

We performed a detailed analysis of patient demographics, clinical presentation, radiological findings, surgical management and clinical outcome.

Post-operative patient review was performed on a six-monthly basis, with the position of the orbital floor being monitored using nasendoscopy and clinical measurement of enophthalmos. Follow-up patients underwent computed tomography scanning of the paranasal sinuses only if clinically indicated. Our follow-up period ranged from 15 months to 10 years.

\section{Results and analysis}

We have managed 23 cases of chronic maxillary atelectasis in our unit, of which 18 had clinical evidence of silent sinus syndrome. Fourteen of these patients were female and nine were male, giving a male:female ratio of 1:1.5. Patients' ages ranged from 19 to 54 years at the time of diagnosis. The right maxillary sinus was affected in 15 cases, the left in eight cases.

\section{Clinical presentation}

Seven patients presented for investigation of progressive facial asymmetry and clinical enophthalmos. The remainder were referred following incidental radiological findings.

On direct questioning, six patients confirmed symptoms of nasal obstruction and/or snoring. One patient with a significant septal deviation reported a history of recurrent epistaxis. Such symptoms were experienced over a period of time ranging from months to a few years, but were not experienced by those with clinical enophthalmos with silent sinus syndrome. Eighteen patients were found to have evidence of clinical enophthalmos, and seven had obvious facial asymmetry (Figure 1).

\section{Radiological findings}

All patients had radiological evidence of a hypoplastic maxillary antrum with associated opacification. Furthermore, in each case the uncinate process was atelectatic and loosely adherent to the lamina papyracea. Eleven patients also had a deviated nasal septum, with a bony spur to the side of the hypoplastic maxillary sinus.

\section{Management}

All patients underwent endoscopic uncinectomy and maxillary antrostomy (Figure 2), which was combined with a septoplasty in 11 patients. Orbital floor reconstruction was not performed in any of the patients with enophthalmos.

\section{Outcome}

Patients were followed up for a period ranging from 15 months to 10 years.

In this time period, one of the 23 patients had evidence of persistent clinical enophthalmos, at 18 months. In this patient, follow up with nasendoscopy demonstrated elevation of the orbital floor over time. However, he continued to have clinical features of enophthalmos, and consequently was considered to possibly require orbital floor reconstruction.

All patients were reviewed at routine intervals. There was no ongoing evidence of worsening enophthalmos or hypoglobus. Fourteen of the 18 patients with enophthalmos (78 per cent) had complete resolution of enophthalmos, while three (17 per cent) had partial resolution. Partial resolution was defined as some residual enophthalmos, but of no aesthetic concern to the patient. The time period over which improvement in enophthalmos became evident was up to 18 months post-surgery, with changes generally starting to appear from six months post-operatively.

None of the five chronic maxillary sinus atelectasis patients progressed to develop enophthalmos and silent sinus syndrome following sinus re-ventilation, during their follow-up period. 


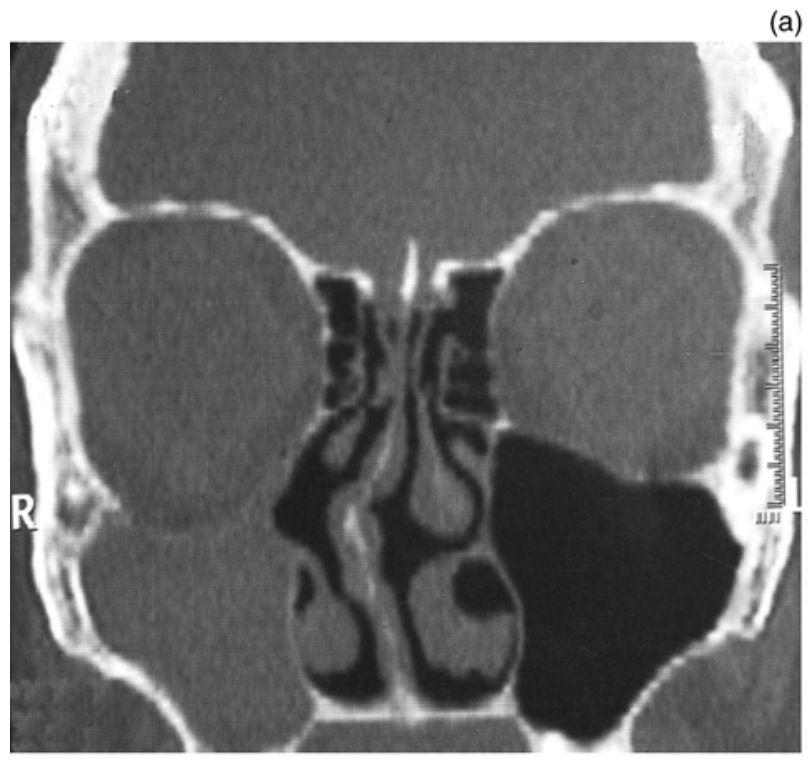

(b)

(a)

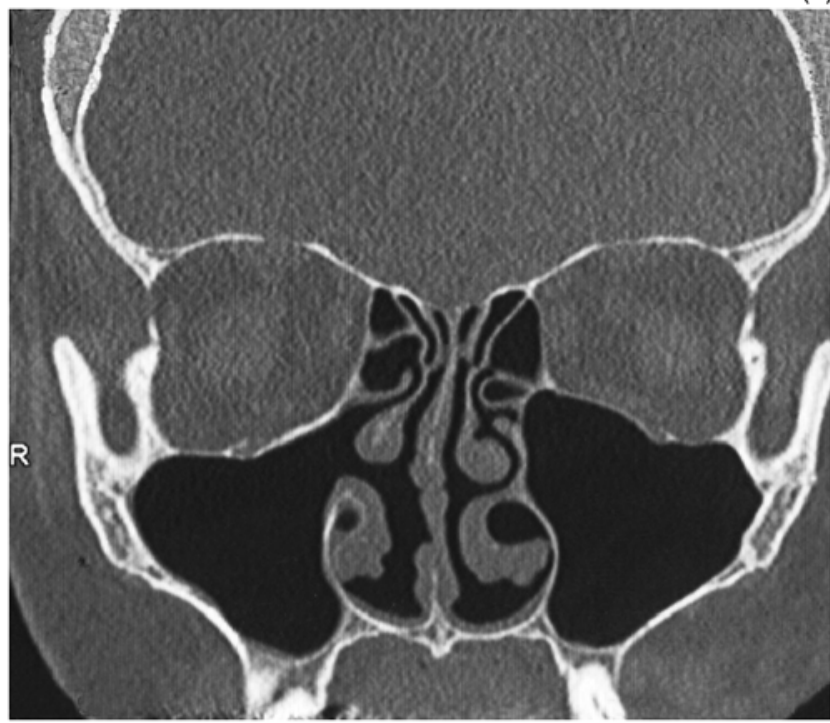

(c)
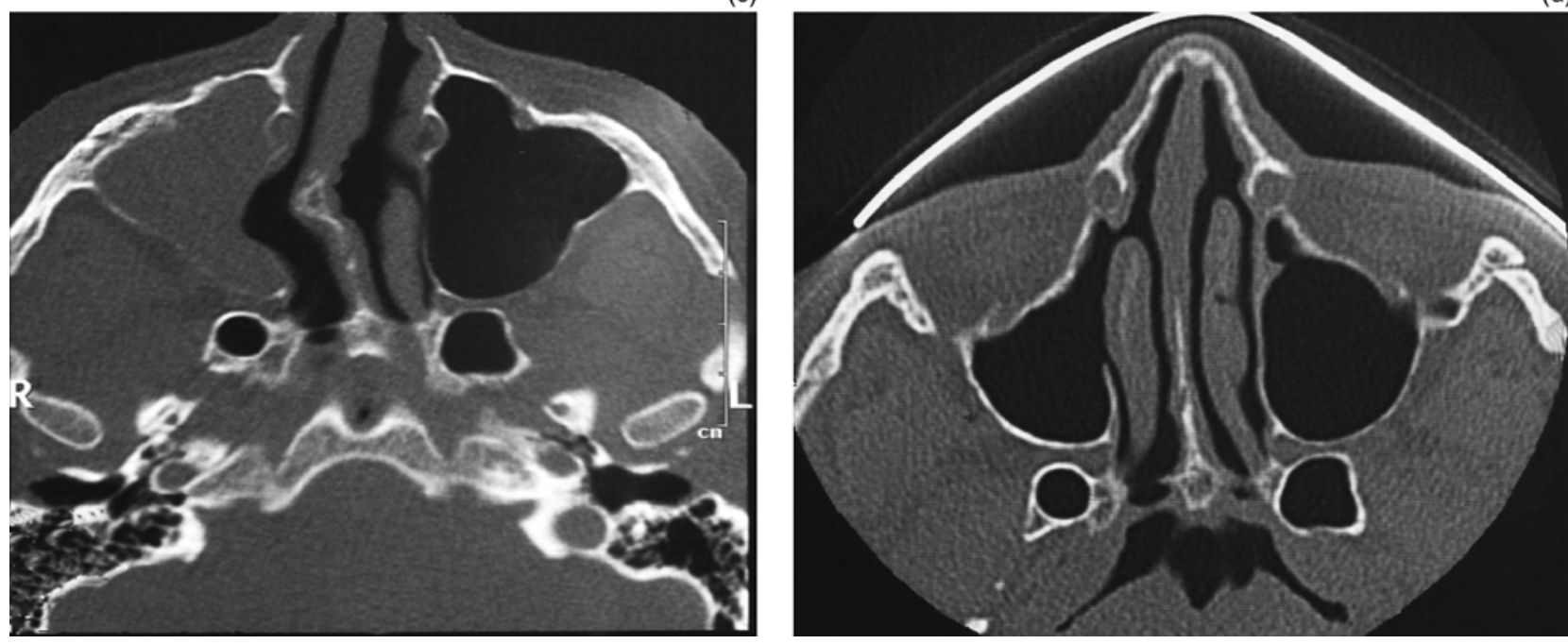

FIG. 1

(a) Coronal and (b) following uncinectomy and middle meatal antrostomy, parts (c) axial computed tomography views of a hypoplastic right maxillary antrum in a patient with silent sinus syndrome and (d) show corresponding views indicating remodelling of the orbital floor and orbital medial wall, 10 years post-surgery.

\section{Discussion}

For years, the management of enophthalmos in silent sinus syndrome patients has included the reestablishment of sinus ventilation by endoscopic uncinectomy and antrostomy combined with simultaneous transconjunctival repair of the orbital floor. ${ }^{9}$ However, there have been recent reports documenting the return of the globe to its normal position after sinus re-ventilation procedures, first by Wan et al. (one case) in 2000 and later by Thomas et al. (two cases) in 2003. ${ }^{10,11}$ A number of review articles published in recent years have suggested the idea of delayed or staged orbital floor correction. ${ }^{12}$ However, there are no studies, besides the above-mentioned ones, which clearly indicate that silent sinus syndrome should be initially treated by uncinectomy and antrostomy alone.
In our unit, all patients with silent sinus syndrome are followed up after sinus surgery, and the position of the orbital floor is monitored. In the presented silent sinus syndrome patients, dynamic changes in the position of the orbital floor were clearly evident, and were documented, at various time points following sinus re-ventilation.

These findings further support the need for a delay before proceeding with reconstruction of the orbital floor, as management of enophthalmos. At the time of writing, none of our subjects had required orbital floor reconstruction, although one patient may require such intervention in the future.

We would assume that the elimination of negative maxillary sinus pressure (demonstrated by Scharf et al.) following endoscopic uncinectomy and antrostomy removes the downward retractive pull on the 

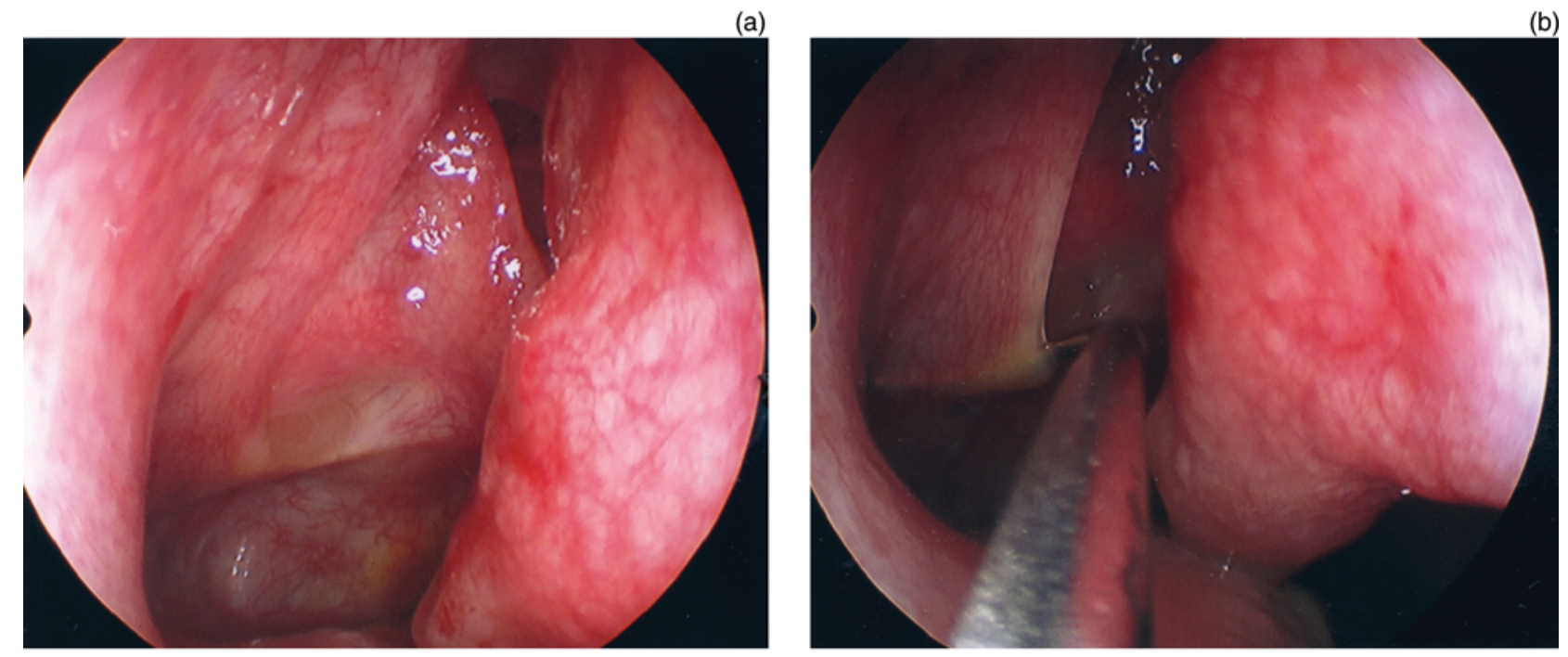

(c)
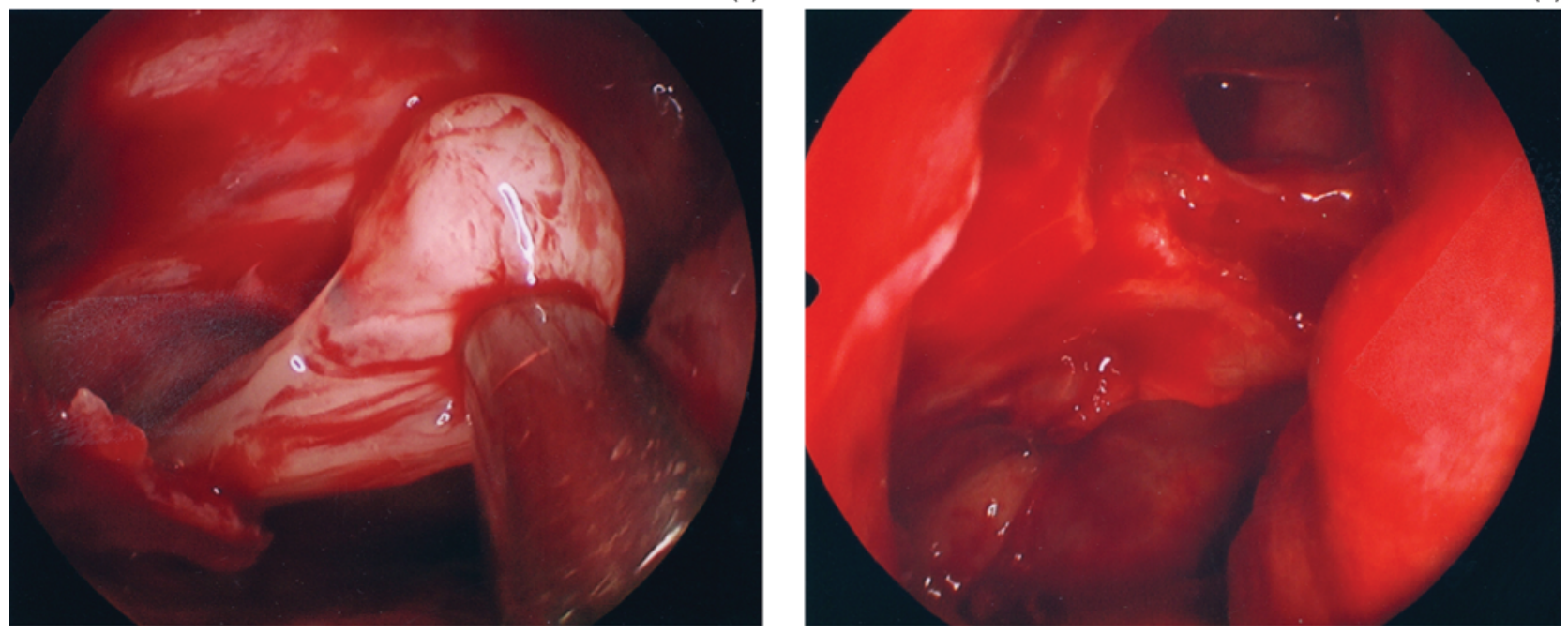

FIG. 2

Intra-operative, endoscopic images showing the uncinectomy and middle meatal antrostomy procedures.

orbital floor and contents, allowing a return to a normal or near-normal position. ${ }^{13}$

- Silent sinus syndrome comprises spontaneous enophthalmos and hypoglobus, with chronic maxillary sinus atelectasis and without intrinsic sinonasal inflammatory disease

- This slow, progressive, unilateral disorder eventually causes $2-6 \mathrm{~mm}$ of hypoglobus and 2-5 mm of enophthalmos

- Endoscopic uncinectomy and antrostomy reestablish maxillary sinus ventilation, which usually corrects the orbital floor deformity, reversing enophthalmos and hypoglobus

- If there is no post-operative improvement, orbital floor reconstruction is recommended

In the past, re-establishment of normal maxillary sinus configuration has been demonstrated with imaging; however, this has not been demonstrated in all cases of silent sinus syndrome. The current study demonstrates that it is possible to monitor the post-operative position of the orbital floor with nasendoscopy. In addition to providing a simple means of monitoring, such nasendoscopy also enables assessment of the need for future reconstructive surgery. We performed computed tomography scanning in only two cases post-operatively; in both these cases, there was evidence of bony remodelling, restoring the orbital floor to a normal position.

As previously documented, the risk of orbital injury in such cases is high, because of the close adherence of the uncinate process to the lamina papyracea. ${ }^{14}$ Consequently, we believe that it is essential to perform uncinectomy in a posterior-to-anterior direction, using a paediatric back-biting instrument, to avoid injury to the lamina papyracea (as opposed to using an anteriorto-posterior direction and a sickle knife).

In many cases, an associated septoplasty procedure may be necessary. This was the case in our series of 
patients with chronic maxillary atelectasis and silent sinus syndrome, in which 11 out of 23 patients had significant associated nasal septal deviation.

\section{Conclusion}

In patients with silent sinus syndrome and enophthalmos, the position of the orbital floor can improve following surgical restoration of normal maxillary sinus pressure. Therefore, orbital floor reconstruction, performed to treat enophthalmos in such patients, should be delayed for anything up to 18 months after sinus surgery. In many cases, uncinectomy and antrostomy may be sufficient management for silent sinus syndrome.

In such cases, the close adherence of the uncinate process to the lamina papyracea favours performance of the uncinectomy in a posterior-to-anterior direction.

\section{References}

1 Montgomery WW. Mucocele of the maxillary sinus causing enophthalmos. Eye Ear Nose Throat Mon 1964;43:41-4

2 Soparker CN, Patrinely JR, Cuaycong MJ, Dailey RA, Kersten RC, Rubin PA et al. The silent sinus syndrome: a cause of spontaneous enophthalmos. Ophthalmology 1994;101:772-8

3 Kass ES, Salman S, Montgomery W. Manometric study of complete ostial occlusion in chronic maxillary atelectasis. Laryngoscope 1996;106:1255-8

4 Davidson JK, Soparkar CN, Williams JB, Patrinely JR. Negative sinus pressure and normal predisease imaging in silent sinus syndrome. Arch Ophthalmol 1999;117:1653-4

5 Kass ES. The diagnosis and treatment of chronic maxillary atelectasis in adults and children. Curr Opin Otolaryngol Head Neck Surg 1999;7:39-45
6 Annino DJ Jr, Goguen LA. Silent sinus syndrome. Curr Opin Otolaryngol Head Neck Surg 2008;16:22-5

7 Brandt MG, Wright ED. The silent sinus syndrome is a form of chronic maxillary atelectasis: a systematic review of all reported cases. Am J Rhinol 2008;22:68-73

8 Dailey RA, Cohen JL. Surgical repair of the silent sinus syndrome. Ophthal Plast Reconstr Surg 1995;11:261-8

9 Vander Meer JB, Harris G, Toohill RJ, Smith TL. The silent sinus syndrome: a case series and literature review. Laryngoscope 2001;111:975-8

10 Thomas RD, Graham SM, Carter KD, Nerad JA. Management of the orbital floor in silent sinus syndrome. Am J Rhinol 2003; 17:97-100

11 Wan MK, Francis IC, Carter PR, Griffits R, van Rooijen ML, Coroneo MT. The spectrum of presentation of silent sinus syndrome. J Neuroophthalmol 2000;20:207-12

12 Numa WA, Desai U, Gold DR, Heher KL, Annino DJ. Silent sinus syndrome: a case presentation and comprehensive review of all 84 reported cases. Ann Otol Rhinol Laryngol 2005;114: 688-94

13 Schraf KE, Lawson W, Shapiro JM, Gannon PJ. Pressure measurements in the normal and occluded rabbit maxillary sinus. Laryngoscope 1995;105:570-4

14 Hunt SM, Tami TA. Sinusitis-induced enopthalmos: the silent sinus syndrome. Ear Nose Throat J 2000;79:578-84

Address for correspondence:

A/Prof R Sacks,

ENT Centre,

Suite 12 The Madison, 25-29 Hunter Street, Hornsby, NSW, Australia 2077

Fax: + 61294824695

E-mail: rsacks@commander360.com

Associate Professor R Sacks takes responsibility for the integrity of the content of the paper

Competing interests: None declared 